\title{
Description of pharmacists' reported interventions to prevent prescribing errors among in hospital inpatients: a cross sectional retrospective study
}

Abdulhakim A. Alzahrani ${ }^{1,2}$, Monira M. Alwhaibi ${ }^{3,4}$, Yousif A. Asiri ${ }^{1,3,4}$, Khalid M. Kamal ${ }^{5}$ and Tariq M. Alhawassi ${ }^{3,4,6^{*}}$

\begin{abstract}
Background: Prescribing errors (PES) are a common cause of morbidity and mortality, both in community practice and in hospitals. Pharmacists have an essential role in minimizing and preventing PEs, thus, there is a need to document the nature of pharmacists' interventions to prevent PEs. The purpose of this study was to describe reported interventions conducted by pharmacists to prevent or minimize PEs in a tertiary care hospital.

Methods: A retrospective analysis of the electronic medical records data was conducted to identify pharmacists' interventions related to reported PEs. The PE-related data was extracted for a period of six-month (April to September 2017) and comprised of patient demographics, medication-related information, and the different interventions conducted by the pharmacists. The study was carried in a tertiary care hospital in Riyadh region. The study was ethically reviewed and approved by the hospital IRB committee. Descriptive analyses were appropriately conducted using the IBM SPSS Statistics.

Results: A total of 2,564 pharmacists' interventions related to PEs were recorded. These interventions were reported in 1,565 patients. Wrong dose $(54.3 \%)$ and unauthorized prescription (21.9\%) were the most commonly encountered PEs. Anti-infectives for systemic use (49.2\%) and alimentary tract and metabolism medications (18.2\%) were the most common classes involved with PEs. The most commonly reported pharmacists' interventions were dose adjustments (44.0\%), restricted medication approvals (21.9\%), and therapeutic duplications (11\%).

Conclusions: In this study, PEs occurred commonly and pharmacists' interventions were critical in preventing possible medication related harm to patients. Care coordination and prioritizing patient safety through quality improvement initiatives at all levels of the health care system can play a key role in this quality improvement drive. Future studies should evaluate the impact of pharmacists' interventions on patient outcomes.
\end{abstract}

Keywords: Prescribing errors, Pharmacists related interventions, Acute care setting, Patient safety

\footnotetext{
* Correspondence: tarriq@ksu.edu.sa

${ }^{3}$ Department of Clinical Pharmacy, College of Pharmacy, King Saud University, PO Box 2457, Office (1A229), 11451 Riyadh, Saudi Arabia ${ }^{4}$ Medication Safety Research Chair, College of Pharmacy, King Saud University, Riyadh, Saudi Arabia

Full list of author information is available at the end of the article
}

C C The Author(s). 2021 Open Access This article is licensed under a Creative Commons Attribution 4.0 International License, which permits use, sharing, adaptation, distribution and reproduction in any medium or format, as long as you give appropriate credit to the original author(s) and the source, provide a link to the Creative Commons licence, and indicate if changes were made. The images or other third party material in this article are included in the article's Creative Commons licence, unless indicated otherwise in a credit line to the material. If material is not included in the article's Creative Commons licence and your intended use is not permitted by statutory regulation or exceeds the permitted use, you will need to obtain permission directly from the copyright holder. To view a copy of this licence, visit http://creativecommons.org/licenses/by/4.0/. The Creative Commons Public Domain Dedication waiver (http://creativecommons.org/publicdomain/zero/1.0/) applies to the data made available in this article, unless otherwise stated in a credit line to the data. 


\section{Background}

The benefits of medications in relation to patient care have been one of the main focuses of the human being for centuries. Nowadays, with the obvious advances in medical science, medicines have well-known documented evidence based benefits in terms of effectively treating, managing or preventing various diseases [1]. However, medications can be a double-edged sword and medication safety has always been a major concern for both patients and healthcare professionals (HCPs) [2]. Moreover, with the growth in fast-track drug approvals in the last years coupled with the increasingly complex drug molecules and therapy regimens such as the use of biologics and other sensitive medications, drug-related complications are expected to be on the rise and require more than usual attention from all HCPs [3].

Prescribing errors (PEs), a major medication safety issue, are a common cause of morbidity and mortality, both in the community practice and in hospitals [4]. PEs are defined as "a clinically meaningful prescribing error that occurs as a result of a prescribing decision or the prescription writing process resulting in an unintentional significant reduction in the probability of treatment being timely and effective [5] or in increasing the risk of harm when compared to generally accepted practice" [6]. Despite the fact that there is variability in the documented rates of medication errors due to the utilization of various medication safety classification systems in addition to the different tools and methods of recording medication errors, PEs are nevertheless considered a common occurrence with substantially high burden [7]. Moreover, PEs are associated with higher consumption of healthcare resources and incur substantial costs to the healthcare system [8].

The occurrence of PEs in hospitals is a perennial problem and can occur at any stage of the medication process. The incidence of PEs in patient care, in part, can be attributed to the quality of care that is provided and also to the complexity of the drug regimen that is prescribed to the patient. Almost $6.5 \%$ of morbidity and mortality in hospitalized patients have been linked to PEs, while more than half of these errors are considered as preventable [9]. Published studies have reported that PEs can also occur due to prescribing wrong medications or doses, wrong frequency, duplicate orders, and prescription of restricted medications. This problem is further compounded in critically ill patients in acute care settings who are at an increased risk for PEs given their severe health status, treatment with complex regimen, polypharmacy and constant treatment modifications [10]. Additionally, the high frequency of PEs in hospitals can also be attributed to hospital system such as inadequate medical staff training, increased work load or secondary to patient-related factors such as poor patient health literacy in addition to other various factors such as the similarly sounding medication names, and etcetera [11].

Pharmacists are considered one of the main key players in the healthcare system with other HCPs working in a multidisciplinary approach [12]. Therefore, pharmacists have an essential role in almost all medication processes and can positively impact patient care and patient safety [13]. This role can be seen in the different settings including hospital multidisciplinary teams, nursing homes as well as in the primary care settings. In terms of medication and patient safety, for instance, some studies have reported that about threequarters of PEs can be addressed by pharmacists before reaching patients $[14,15]$. Consequently, pharmacist's role in the optimization of drug therapy and medication management, from a patient safety and quality of care point of view has always to be quantified by the level of interventions provided by pharmacists to prevent medications misadventures in addition to the other services that pharmacists provide [16].

With the advances in the pharmaceutical care filed, pharmacists have developed different patient safety approaches and have played an important role in the application of these approaches in the healthcare system to improve patient safety [17]. Their range of roles differ widely, from increasing patient knowledge through drug information or teaching patients to correctly use the medications so as to prevent adverse drug events (ADEs) during inpatient stay or post hospital discharge $[17,18]$. In a clinical trial conducted in the critical care setting, the involvement of a pharmacist in patient care was associated with three- to five-fold reduction in the incidence of PEs [19]. Searching the literature shows a very few studies have addressed the nature of pharmacists' related interventions to prevent PEs in tertiary care settings and in Saudi Arabia this issue is not well reported and thus, there is a need to explore the different interventions provided by pharmacists to minimize or prevent PEs to assess its impact on patient outcomes and pave the way for future studies that can implement the various pharmacists' interventions and its impact on patient outcomes [20].

\section{Aim of study}

The main aim of this study was to describe the different pharmacists delivered interventions to prevent or minimize PEs among inpatients in a hospital setting.

\section{Ethics approval}

Ethics approval was obtained for this project through King Saud University Institution Review Board. Approval was granted on May 21, 2018: approval number [E-18-3251]. 


\section{Methods}

\section{Study Design and setting}

A retrospective cross-sectional analysis of the electronic medical records (EMRs) system of a tertiary teaching hospital was conducted to identify reported pharmacists' interventions related to PEs. The hospital is one of the leading tertiary teaching hospitals in Riyadh, Saudi Arabia with a capacity of over 1,700 beds and more than 40 medical and surgical wards. The hospital approximate number of admissions is 50,000 new inpatients per year.

\section{Sample size and study period}

Patients of all ages who were admitted to the inpatient care and prescribed/received a medication during the study period and had complete report and documented PEs and pharmacists' reported interventions during the study period (April 2017 to September 2017) were included in this study. The PEs related data of all medical, surgical wards, or other wards patients was extracted. Patients from the outpatient setting or those in an inpatient setting with incomplete reported PEs and pharmacists' interventions were excluded.

\section{PEs and Pharmacists Interventions Reporting process}

The Electronic System for Integrated Health Information (eSiHi application) is an electronic system that allows HCPs in the study site to report all patients related data (medical, laboratory and pharmacy related data) including any medical incidents which include medication errors (MEs) data, allergies, etc...). The hospital time frame for reporting any potential MEs is $24 \mathrm{~h}$ for significant/sentinel event and $72 \mathrm{~h}$ for non-sentinel events. The data pertaining to any potential MEs to be reported includes clinical data, medication information and data related to MEs including PEs obtained by the clinical pharmacists in the different medical, surgical and other inpatients wards at the time of patient hospital admission or discharge. This part of the reporting process using the ESiHi is defined and known as the "Intervention Report". Afterwards, once the reported PEs issue been solved by the medical team and the proper decision is taken with error got corrected, the Medication Safety Officer (MSO) who works under the hospital's department of pharmaceutical care services (Quality and Safety Unit)traces the reports from the time received until the reported MEs get evaluated and analyzed in the system. Furthermore, to ensure accuracy of reporting, the patients' medical record is requested and the reporter can be contacted for any clarification, when completed MEs reports get assessed and verified by the in charge MSO. A quarterly MEs analysis and report is presented to the Pharmacy and Therapeutic Committee for review and appropriate measures. This practice is performed for quality assessment to assure safe medical practice and therefore patient safety, according to the institute policy and procedures and proper actions which act hard to improve the "Just Culture" environment in addition to reporting medical related problems to the Saudi Food and Drug Authority Vigilance Department if required. The Quality and Safety Unit has an effective and consistent policy on how to handle MEs. Moreover, it gives appropriate instructions and precautions to clinical pharmacists on how to identify, report, intervene, and analyze medication errors. Also, it has a system in place for monitoring, and preventing future incidences. MSOs are highly trained with high education and training in pharmacy and medication and patient safety.

\section{Data Collection}

All data pertaining to PEs and pharmacists' reported interventions "Intervention Report" and data related to medication use was extracted retrospectively from ESiHi. These reports are either done by the pharmacist-incharge of the hospital wards or in the central inpatient pharmacy, which is then retrieved by the MSO at the study site for confirmation and auditing. The extracted data was then entered by the study main investigator (AA) in an excel file designed purposefully for the study. The extracted data was cleaned, reviewed, and validated by the study co-investigator (TA). Other study team members (MA and YA) were consulted for verification or for addressing conflicting reports. The final dataset comprised of patient demographics (age, gender, and nationality), medication-related information, PEs data, and the nature of interventions performed by the pharmacists. Patients were classified into five age groups: infants ( 0 to 2 years), children ( 3 to 12 years), adolescents (13 to 17 years), adults (18 to 64 years) and older adults (65 years and higher).

\section{PEs Definition, categorization and pharmacists related interventions}

All the PEs and comments written in the Intervention Report were reviewed and classified into 11 categories according to the hospital classification system which defines PEs as: (i) prescribing of wrong dose, (ii) prescribing of wrong medication, (iii) prescribing of duplicate therapy, (iv) prescribing of contraindicated drugs, (v) prescribing medication to patient with documented Adverse Drug Event (ADR) or Allergy, (vi) prescribing of wrong time of administration or rate, (vii) prescribing of wrong route, (viii) prescribing of wrong frequency, (ix) incomplete prescription or lack of other information, $(\mathrm{x})$ unauthorized prescription (prescription of medications restricted to certain specialty by unauthorized prescriber), (xi) others.

Pharmacists' interventions were classified into 12 different intervention types according to the hospital 
system: (i) preventing an adverse drug event, (ii) stopping contraindicated medications, (iii) dose adjustments, (iv) preventing drug interactions, (v) conversions from intravenous to oral formulations, (vi) discontinuation of medications with no indications, (vii) optimizing therapy monitoring (i.e. including therapeutic drug monitoring, prescriptions information quality (incomplete prescription), wrong dose and wrong frequency etc...), (viii) renal dose adjustments, (ix) approval of restricted medications, ( $\mathrm{x}$ ) therapeutic duplications prevention, (xi) recommending medications for untreated indications, (xii) others unclassified interventions.

\section{Medications and route of Administration classification}

The medications reported in this study were classified based on the Anatomical Therapeutic Chemical (ATC) classification system. The route of administration for medications was divided into two main groups: systemic (enteral and parenteral medications) and local (topical and inhalation medications).

\section{Statistical analysis}

Descriptive analyses were conducted using IBM SPSS Statistics (Version 21, Corp, Armonk, NY, USA). Frequency and percentage were used to describe the categorical variables. Mean and Standard deviation were used to describe continuous variables.

\section{Results}

\section{Occurrence and Types of PEs}

A total of 2,564 PEs and pharmacists' interventions related to PEs among 1,565 patients were recorded in the hospital database during the study period. Patients' age ranged from 1 to 104 years, with a mean age $( \pm S D)$ of $42.72( \pm 27.22)$ years. The highest frequency of reported PEs was among adults (18 to 64 years) had with 1,241 (48.4\%) reported PEs, while the lowest frequency of PEs reported (4.3\%) was among adolescents' patients (13 to 17 years). PEs among other ages were also noticeable with 253 (9.9\%) PEs in infants (0 to 2 years), 257 $(10.0 \%)$ of PEs in children (3 to 12 years) and 704 $(27.5 \%)$ occurred in older patients ( $\geq 65$ years). The reported PEs were higher in females $(n=1,320,51.5 \%)$ and among Saudi citizens $(n=2,117,82.6 \%)$ compared to males and non-Saudi, respectively. The highest rate of PEs was recorded in the medical wards followed by intensive care units (Table 1).

The highest rate of PEs was for prescribing wrong doses $(n=1,393,54.3 \%)$ followed by PEs related to unauthorized prescriptions $(n=562,21.9 \%)$ (Table 2). Anti-infectives for systemic use and alimentary tract and metabolism were the two most common medications associated with the reported PEs (Table 3). Medications for systemic use and in particular parenteral medications
Table 1 Distribution of prescription errors according to the different hospital's units ${ }^{\mathrm{a}}(n=2564)$

\begin{tabular}{lll}
\hline & \multicolumn{2}{l}{ Prescription Errors } \\
\cline { 2 - 3 } & No. & $\%$ \\
\hline Medical wards & 1,554 & 60.6 \\
Hemodialysis Unit & 45 & 1.8 \\
Hospital Isolation Rooms & 90 & 3.5 \\
Academic Staff & 26 & 1.0 \\
Business Center & 57 & 2.2 \\
Cardiac Medical Ward & 38 & 1.5 \\
Emergency Department & 205 & 8.0 \\
Internal Medicine Ward & 594 & 23.2 \\
Nursery Unit & 8 & 0.3 \\
Obstetrics and Gynecology & 132 & 5.1 \\
Oncology & 44 & 1.7 \\
Pediatric & 290 & 11.3 \\
Psychiatric Ward & 25 & 1.0 \\
Intensive Care Unit & 614 & 23.9 \\
Surgical Unit & 390 & 15.2 \\
Radiology Department & 6 & 0.2 \\
\hline
\end{tabular}

${ }^{a}$ Hospital units classified according to the hospital internal system as listed in this table.

had the highest rate of PEs that required pharmacists' interventions (Table 3).

\section{PEs and Pharmacists' Interventions}

Pharmacists' interventions to prevent PEs were categorized into 12 types. The highest observed pharmacists related interventions to prevent PEs were related to dose adjustments $(n=1,128,44 \%)$, followed by approval of restricted medications $(n=562,21.9 \%)$, prevention of therapeutic duplication $(n=281,11.0 \%)$, renal patients dosing ( $n=249,9.2 \%)$, and optimizing therapy monitoring $(n=179, \% 6.8$ (Table 4).

\section{Discussion}

The present study retrospectively identified PEs and the different pharmacist's related interventions in an inpatient setting in a large tertiary teaching hospital in Saudi Arabia. A total of 2,564 PEs were recorded during a six-month period affecting 1,565 patients in a ratio of 1.7 pharmacist interventions for encountered PEs per a patient.

There is a high burden of PEs on the healthcare system in Saudi Arabia. A systematic review of PEs in Saudi Arabia (both community and hospital settings) reported the PEs rates vary from 7.1 to $94 \%$ with a median error rate of $32 \%$ [21]. Another systematic review evaluating PEs in patients admitted to a hospital found median 
Table 2 Types of Prescribing Errors (PEs) $(n=2564)$

\begin{tabular}{lll}
\hline Variables & \multicolumn{2}{l}{ Types of PEs } \\
\cline { 2 - 3 } & No. & (\%) \\
\hline Prescribing of wrong dose & 1,393 & 54.3 \\
Unauthorized prescription & 562 & 21.9 \\
Prescribing of duplicate therapy & 279 & 10.9 \\
Prescribing of Contraindicated drugs & 98 & 3.8 \\
Incomplete prescription/ lack of information / & 89 & 3.5 \\
Or require monitoring & & \\
Prescribing of wrong frequency & 58 & 2.3 \\
Prescribing of wrong route & 53 & 2.1 \\
Prescribing of wrong medication & 18 & 0.7 \\
Prescribing of wrong time of administration / Rate & 7 & 0.3 \\
Prescribing medication to patient with documented & 4 & 0.2 \\
ADR/ Allergy & & \\
Failure to monitor when required & 3 & 0.1 \\
\hline ADR Adve Drug Reaction; PEs Prescibing Errs
\end{tabular}

ADR Adverse Drug Reaction; PES Prescribing Errors

Table 3 Medications' therapeutic categories associated with PEs using ATC Classification ( $n=2,564)$

\begin{tabular}{|c|c|c|}
\hline Medications' Therapeutic Categories & $\mathrm{N}$ & $\%$ \\
\hline Alimentary tract and metabolism (A) & 466 & 18.2 \\
\hline Anti infectives for systemic use $(J)$ & 1,261 & 49.2 \\
\hline Antineoplastic and immune modulating agents $(\mathrm{L})$ & 16 & 0.6 \\
\hline Blood and blood forming organs (B) & 267 & 10.4 \\
\hline Cardiovascular system (C) & 142 & 5.5 \\
\hline Dermatologicals (D) & 38 & 1.5 \\
\hline Genitourinary system and Sex hormones (G) & 8 & 0.3 \\
\hline Musculoskeletal system (M) & 51 & 2.0 \\
\hline Nervous system (N) & 216 & 8.4 \\
\hline Pituitary, hypothalamic hormones, systemic hormonal & 23 & 0.9 \\
\hline \multicolumn{3}{|l|}{ preparations, and insulin analogues $(\mathrm{H})$} \\
\hline Respiratory System (R ) & 71 & 2.8 \\
\hline Sensory Organs (S) & 4 & 0.2 \\
\hline Various (V) & 1 & 0.1 \\
\hline \multicolumn{3}{|l|}{ PEs according to Route of Administration } \\
\hline \multicolumn{3}{|l|}{ Systematic } \\
\hline Enteral & & 40.5 \\
\hline Parenteral & & 58.0 \\
\hline \multicolumn{3}{|l|}{ Local } \\
\hline Topical & & 0.2 \\
\hline Inhalation & & 0.9 \\
\hline
\end{tabular}

ATC The Anatomical Therapeutic Chemical (ATC) Classification System; PEs Prescribing Errors
Table 4 Types of Pharmacists Interventions Related to PEs $(n=2564)$

\begin{tabular}{lll}
\hline Type of Intervention & No. & (\%) \\
\hline Dose adjustment & 1,128 & 44.0 \\
Restricted medication approval & 562 & 21.9 \\
Therapeutic duplication & 281 & 11.0 \\
Renal dosing interventions & 249 & 9.7 \\
Optimize therapy monitoring & 174 & 6.8 \\
IV to PO conversion ${ }^{\text {a }}$ & 53 & 2.1 \\
Contraindicated drugs & 48 & 1.9 \\
Drug interaction & 46 & 1.8 \\
Untreated Indications & 9 & 0.4 \\
No indication for therapy & 8 & 0.3 \\
Adverse Drug Event & 4 & 0.2 \\
Others & 2 & 0.1 \\
\hline a & &
\end{tabular}

aV Intravenous; PEs Prescribing Errors; PO Oral

rates of 52 PEs per 100 admissions and 24 PEs per 1,000 patient days [22]. There seem to be a huge discrepancy in the prevalence of error rates including their method of estimation. This is mainly due to variability in study settings, techniques used to detect PEs [23], and also the variability in PEs definitions applied by the different studies [24]. This could potentially explain the difference in the prevalence of PEs recorded in Europe compared to those in the Middle East [25]. Therefore, there has been a growing interest in the PEs area in terms of standardizing the definitions of PEs encountered in Saudi Arabia hospitals. As a result, a recently published study tried to operationalize the different PEs in the Saudi Arabia hospital practice [24]. The availability of a standardized list of PEs to the pharmacists similar to the one applied by the study site may improve their participation in shared decision-making before medications are administered to patients in the acute care units [26]. It can also serve as a valuable resource for any future research related to PEs [24].

The present study showed that the PEs was more common in the medical wards as compared to the surgical ward. This is has been noticed in other studies which have explained the higher rate PEs in medical wards due to the higher proportion of drug prescribing being on admission [27]. In this study, pharmacists recorded 12 different type of PEs, where most of the PEs recorded were related to prescribing wrong doses. Also it is worth mentioning that among the reported PEs unauthorized prescription was fairly high and ranked second after prescribing of wrong dose representing half of the whole errors in the study population [7]. This is consistent with the findings of another study conducted in a university hospital in Riyadh, Saudi Arabia in 2011, which reported that the leading PEs were associated with wrong dose 
and wrong strength [28]. Our finding is consistent with a study that was conducted in the inpatients setting in a teaching hospital in UK which reported that most of the prescribing errors (54\%) were associated with choice of dose [23]. A systematic review of nine studies that involved 3,507 records reported that the top type of prescribing errors was wrong dose which ranged between 31 and $91 \%$ [22]. It is worth to mention that among the different age groups, adult patients experienced the highest PEs rate. This finding is not consistent with other published studies which found that the most affected group with PEs were elderly patients of age 65 and older $[29,30]$. The difference could be attributed to the larger range of adults group included in our study, which included both middle age and young adults which reflect the dominance of young adults in Saudi Arabia where approximately two-third the population age between 15 and 64 years.

The pharmacists' interventions for adjusting doses were the most prevalent intervention followed by restricted medication approval where the most commonly involved class of medications associated with these interventions were anti-infectives for systemic use and alimentary tract and metabolism. Surprisingly, this study reported that only $0.2 \%$ of the pharmacists interventions were related to ADEs and this finding was in contrast with those reported by the Harvard Medical Practice Study which reported that $19 \%$ of the interventions are related to adverse events in hospitals and emergency department settings [31]. This difference can be attributed to the fact that the Harvard study was conducted in an emergency department, where most of ADEs were detected [19], while the present study included all patients admitted to the hospitals. Despite the different interventions required by pharmacists to prevent PEs, current evidence demonstrate that hospital pharmacist's participation in inpatient care can result in significant decrease of avoidable PEs [23]. Yet, limited information is available on pharmacists' interventions within the inpatient setting, especially in Saudi Arabia [26, 28]. A metaanalysis of four controlled trials investigating the impact of pharmacist's interventions on PEs revealed that pharmacists' interventions have a significant impact in reducing PEs [32]. This study results were consistent with the findings reported in the meta-analysis in terms of reduction avoidable PEs.

This study investigated the classes of medications as it related to the incidence of PEs. It was found that systemic use of anti-infective agents was a leading reason for PEs with almost half the errors belonged to this class. This finding is consistent with a recent data supporting that PEs were mostly associated with the orders for antibiotics [33], while other drugs such as antiepileptics, had less frequent errors. Another study conducted in 20 hospitals in UK that included 26,019 prescriptions show that the most of PEs were in parenteral administration of drugs, however, they reported that cardiovascular and endocrine medications were the most common classes associated with PEs [34].

This study utilized a large sample size, and tried to investigate an important research question that can lead to future research to improve the current pharmacy practice towards safer medication use and preventing or minimizing PEs among hospital setting patients. Nevertheless, there are some inherent limitations in this study. First, although the study was conducted in a single tertiary care hospital that is considered one of the largest hospitals nationally, yet this may limit the generalizability of the study results to other practices within Saudi Arabia making the need for future multicenter studies that included different hospitals with different practices is warranted. Second, the PEs were extracted from the medical records and it is possible that some PEs may not have been recorded therefore future prospective approaches worthy to be conducted. This also necessitate the conduction of future studies to evaluate the prevalence of PEs and therefore the impact of pharmacists interventions on reducing PEs and medications related adverse events from reaching patients. Finally, both the PEs and pharmacists' interventions were operationalized for the study, and yet, there may be variations among the hospital pharmacists in how they provided their interventions and the quality of reporting. However, this study still provides critical information related to patient safety and the significant role of hospital pharmacists in preventing avoidable PEs from reaching patients.

\section{Conclusions}

In this study, PEs occurred commonly and pharmacists' interventions were critical in preventing possible harm to patients. There is a need for coordination of care among different $\mathrm{HCPs}$ to prioritize patient safety through quality improvement initiatives at all levels of the health care system where pharmacists can play a key role in this quality improvement drive. Future studies should evaluate the impact of pharmacists' interventions on patient outcomes.

\section{Abbreviations \\ ADEs: Adverse Drug Event; ADR: Adverse Drug Reaction; ATC: Anatomical Therapeutic Chemical; ESiHi: Electronic System for integrated Health information; HCPs: Healthcare Professionals; IRB: Institutional Review Board; MSO: Medication Safety Office; PEs: Prescribing Errors; SPSS: Statistical Package for the Social Science}

\section{Acknowledgements}

The authors of this research would like to acknowledge the Vice-Deanship of Research Chairs, King Saud University, Riyadh, Saudi Arabia for their financial support to fund this research.

\section{Authors' contributions}

AA and TA raised awareness of this topic, drafted the initial manuscript and reviewed the final version of the manuscript. AA, TA, and MA conceptualized and designed the manuscript structure, carried out the literature review and 
revised the final version of the manuscript to obtain ethics approval for this study. KK and YA critically reviewed the manuscript including the data for important intellectual content. All authors approved the final manuscript as submitted and agreed to be accountable for all aspects of the work.

\section{Funding}

No funding was obtained.

\section{Availability of data and materials}

The datasets used and/or analyzed during the current study are available from the corresponding author on reasonable request.

\section{Declarations}

\section{Ethics approval and consent to participate}

Ethics approval was obtained for this project through King Saud University Institution Review Board. Approval was granted on May 21, 2018: approval number [E-18-3251]. Administrative permissions from the IRB were required to access and use the ESiHi data/records.

\section{Consent for publication}

The authors consent to publish the manuscript.

\section{Competing interests}

The authors declare that they have no competing interests.

\section{Author details}

${ }^{1}$ College of Pharmacy, Riyadh Elm University, Riyadh, Saudi Arabia. ${ }^{2}$ Pharmaceutical Care Department, King Fahad Hospital, Ministry of Health, Albaha, Saudi Arabia. ${ }^{3}$ Department of Clinical Pharmacy, College of Pharmacy, King Saud University, PO Box 2457, Office (1A229), 11451 Riyadh, Saudi Arabia. ${ }^{4}$ Medication Safety Research Chair, College of Pharmacy, King Saud University, Riyadh, Saudi Arabia. ${ }^{5}$ Division of Pharmaceutical, Social and Administrative Sciences, School of Pharmacy, Duquesne University, 600 Forbes Avenue, PA 15282 Pittsburgh, USA. ${ }^{6}$ Pharmacy Services, King Saud University Medical City, Riyadh, Saudi Arabia.

\section{Received: 11 February 2020 Accepted: 11 October 2020}

Published online: 06 May 2021

\section{References}

1. Armstrong D. The rise of surveillance medicine. Sociol Health IIIn. 1995;17(3): 393-404.

2. Buurma H, De Smet PA, Leufkens HG, Egberts AC. Evaluation of the clinical value of pharmacists' modifications of prescription errors. $\mathrm{Br} \mathrm{J}$ Clin Pharmacol. 2004:58(5):503-11.

3. Tigabu BM, Daba D, Habte B. Drug-related problems among medical ward patients in Jimma university specialized hospital, Southwest Ethiopia. Journal of research in pharmacy practice. 2014;3(1):1.

4. Patel N, Desai M, Shah S, Patel P, Gandhi A. A study of medication errors in a tertiary care hospital. Perspectives in clinical research. 2016;7(4):168.

5. Folli HL, Poole RL, Benitz WE, Russo JC. Medication error prevention by clinical pharmacists in two children's hospitals. Pediatrics. 1987:79(5):718-22.

6. Blum KV, Abel S, Urbanski CJ, Pierce J. Medication error prevention by pharmacists. Am J Hosp Pharm. 1988:45(9):1902-3.

7. Chen YF, Neil KE, Avery AJ, Dewey ME, Johnson C. Prescribing errors and other problems reported by community pharmacists. Ther Clin Risk Manag. 2005;1(4):333-42

8. Johnson J, Booman L. Drug-related morbidity and mortality. Journal of Managed Care Pharmacy. 1996;2(1):39-47.

9. Abdel-Latif MM. Knowledge of healthcare professionals about medication errors in hospitals. J Basic Clin Pharm. 2016:7(3):87-92.

10. Reis WC, Scopel CT, Correr CJ, Andrzejevski VM. Analysis of clinical pharmacist interventions in a tertiary teaching hospital in Brazil. Einstein (Sao Paulo). 2013;11(2):190-6.

11. World Health Organization. Medication Errors: Technical Series on Safer Primary Care. 2016 [Internet]. [cited 2019 Mar 8].

12. Spinewine A, Fialová D, Byrne $\mathrm{S}$. The role of the pharmacist in optimizing pharmacotherapy in older people. Drugs Aging. 2012;29(6):495-510.
13. Keers RN, Williams SD, Cooke J, Ashcroft DM. Prevalence and nature of medication administration errors in health care settings: a systematic review of direct observational evidence. Ann Pharmacother. 2013;47(2):237-56.

14. Kim Y, Schepers G. Pharmacist intervention documentation in US health care systems. Hospital pharmacy. 2003;38(12):1141-7.

15. Al Rahbi HA, Al-Sabri RM, Chitme HR. Interventions by pharmacists in outpatient pharmaceutical care. Saudi Pharm J. 2014;22(2):101-6.

16. Al-jedai A, Qaisi S, Al-meman A. Pharmacy practice and the health care system in Saudi Arabia. The Canadian journal of hospital pharmacy. 2016;69(3):231.

17. Azhar S, Hassali MA, Ibrahim MIM, Ahmad M, Masood I, Shafie AA. The role of pharmacists in developing countries: the current scenario in Pakistan. Human Resources for Health. 2009:7(1):54.

18. Roblek T, Deticek A, Leskovar B, Suskovic S, Horvat M, Belic A, et al. Clinicalpharmacist intervention reduces clinically relevant drug-drug interactions in patients with heart failure: a randomized, double-blind, controlled trial. Int J Cardiol. 2016;203:647-52.

19. Wang T, Benedict N, Olsen KM, Luan R, Zhu X, Zhou N, et al. Effect of critical care pharmacist's intervention on medication errors: A systematic review and meta-analysis of observational studies. J Crit Care. 2015;30(5):1101-6.

20. Dalton K, Byrne S. Role of the pharmacist in reducing healthcare costs: current insights. Integr Pharm Res Pract. 2017:6:37-46.

21. Alhomoud FK, Alnemari W, Alfahmi H, Alhomoud F, Cheema E. Incidence and Prevalence of Prescribing Errors in Saudi Arabia: A Systematic Study. International Journal of Pharmacy and Pharmaceutical Sciences. 2016;8(12)

22. Alanazi MA, Tully MP, Lewis PJ. A systematic review of the prevalence and incidence of prescribing errors with high-risk medicines in hospitals. J Clin Pharm Ther. 2016:41(3):239-45.

23. Dean $B$, Schachter $M$, Vincent $C$, Barber N. Prescribing errors in hospital inpatients: their incidence and clinical significance. Qual Saf Health Care. 2002;11(4):340-4.

24. Assiri GA, Shebl NA, Mahmoud MA, Aloudah N, Grant E, Aljadhey $H$, et al. What is the epidemiology of medication errors, error-related adverse events and risk factors for errors in adults managed in community care contexts? A systematic review of the international literature. BMJ Open. 2018;8(5):e019101.

25. Dean Franklin B, Vincent C, Schachter M, Barber N. The incidence of prescribing errors in hospital inpatients: an overview of the research methods. Drug Saf. 2005;28(10):891-900

26. Leape LL, Brennan TA, Laird N, Lawthers AG, Localio AR, Barnes BA, et al. The nature of adverse events in hospitalized patients: results of the Harvard Medical Practice Study II. New England journal of medicine. 1991;324(6):377-84.

27. Franklin BD, Reynolds M, Shebl NA, Burnett S, Jacklin A. Prescribing errors in hospital inpatients: a three-centre study of their prevalence, types and causes. Postgrad Med J. 2011;87(1033):739-45.

28. Al-Dhawailie AA. Inpatient prescribing errors and pharmacist intervention at a teaching hospital in Saudi Arabia. Saudi Pharm J. 2011;19(3):193-6.

29. Leape LL, Cullen DJ, Clapp MD, Burdick E, Demonaco HJ, Erickson Jl, et al. Pharmacist participation on physician rounds and adverse drug events in the intensive care unit. JAMA. 1999:282(3):267-70.

30. Kozer E, Scolnik D, Macpherson A, Keays T, Shi K, Luk T, et al. Variables associated with medication errors in pediatric emergency medicine. Pediatrics. 2002;110(4):737-42.

31. Mohebbi N, Javadi M, Gholami K. Prescribing error reporting and pharmacist oriented prevention program in emergency department. European Journal of Hospital Pharmacy: Science Practice. 2012;19(2):115-

32. Cunningham KJ. Analysis of clinical interventions and the impact of pediatric pharmacists on medication error prevention in a teaching hospital. J Pediatr Pharmacol Ther. 2012;17(4):365-73.

33. Lucca JM, Ramesh M, Narahari GM, Minaz N. Impact of clinical pharmacist interventions on the cost of drug therapy in intensive care units of a tertiary care teaching hospital. J Pharmacol Pharmacother. 2012;3(3):242-7.

34. Akbarov A, Kontopantelis E, Sperrin M, Stocks SJ, Williams R, Rodgers S, et al. Primary Care Medication Safety Surveillance with Integrated Primary and Secondary Care Electronic Health Records: A Cross-Sectional Study. Drug Saf. 2015:38(7):671-82.

\section{Publisher's Note}

Springer Nature remains neutral with regard to jurisdictional claims in published maps and institutional affiliations. 